\title{
Aggregation Behavior of Some Asymmetric Porphyrins versus Basic Biological Tests Response
}

\author{
Radu Socoteanu, ${ }^{1}$ Mihai Anastasescu, ${ }^{1}$ Anabela Oliveira, ${ }^{2,3}$ Gianina Dobrescu, ${ }^{1}$ \\ Rica Boscencu, ${ }^{4}$ and Carolina Constantin ${ }^{5}$ \\ ${ }^{1}$ Romanian Academy, "Ilie Murgulescu" Institute of Physical Chemistry, 202 Splaiul Independentei, 77208 Bucharest, Romania \\ ${ }^{2}$ Escola Superior de Tecnologia e Gestao, Lugar da Abadessa, 7301-901 Portalegre, Portugal \\ ${ }^{3}$ Centro de Química-Física Molecular e Instituto de Nanociências e Nanotecnologias, Instituto Superior Técnico, \\ Universidade de Lisboa, Avenida Rovisco Pais, 1049-001 Lisboa, Portugal \\ ${ }^{4}$ Faculty of Pharmacy, "Carol Davila” University of Medicine and Pharmacy, Faculty of Pharmacy, 6 Traian Vuia Street, \\ 020956 Bucharest, Romania \\ 5 "Victor Babes" National Institute for Pathology and Biomedical Sciences, 99-101 Splaiul Independentei, 050096 Bucharest, Romania
}

Correspondence should be addressed to Mihai Anastasescu; manastasescu@icf.ro and Anabela Oliveira; asoliveira@estgp.pt

Received 12 August 2015; Accepted 29 September 2015

Academic Editor: Maxim P. Evstigneev

Copyright (C) 2015 Radu Socoteanu et al. This is an open access article distributed under the Creative Commons Attribution License, which permits unrestricted use, distribution, and reproduction in any medium, provided the original work is properly cited.

\begin{abstract}
Fractal analysis of free bases porphyrins was computed on atomic force microscopy (AFM) micrographs using two different methods: the correlation function method and the variable length scale method. The correlation function method provides fractal dimension only for short scale range; results indicate that only few images have fractal properties for short ranges; for the rest of them, no fractal dimension was found using the correlation function method. The variable length scale method occur information for long range scaling. All samples have fractal properties at higher scaling range. For three samples the correlation function method leads to the same fractal dimension as the variable length scale method and scaling ranges for both methods overlap. Results show the necessity to use both methods to describe the fractal properties of $\mathrm{AB}_{3}$ meso-porphyrins that may be used to predict their relative cell localization. In order to emphasize the influence of fractal and textural properties the results regarding their self-similarity and texture/morphology were further compared with their behavior in biological assessment, that is, functionality of some Jurkat cell lines.
\end{abstract}

\section{Introduction}

Porphyrins are more and more involved in "long-chain" studies, with multiple different approaches of the subject, mainly in diagnosis and therapy of cancer, the emerging concept of theranostics, targeting the third generation of photosensitizers. The use of the porphyrin derivatives in biomedical field is a consequence of their ability to generate reactive species [1]. The application domains are continuously expanding $[2,3]$ their use as sensitizers in photodynamic therapy of cancer being today the object of many interdisciplinary studies [4]. Although a large number of mesosubstituted porphyrins were synthesized since the approval of the first photosensitizer for clinical photodynamic therapy [5], there are still improvements to be made in their synthesis [6] and there is still no clear correlation between the mesosubstituents of porphyrins and their selective accumulation and phototoxicity in tumor cells. Recently it has been established that subcellular localization plays a major role in photodynamic efficacy [7].

We consider the balance between symmetry and asymmetry important, regarding the influence of peripheral substituents especially in tissue uptake. This view is supported by the fact that certain changes in the functional groups on a tetrapyrrolic macrocycle, including those attached in mesopositions, influence in significant way the reactivity and mechanism of the porphyrins involved in environmental reactions. Due to the extended electronic system, the porphyrinic behavior is marked by strong tendencies to form large aggregates [8]. Most used methods to evaluate the 


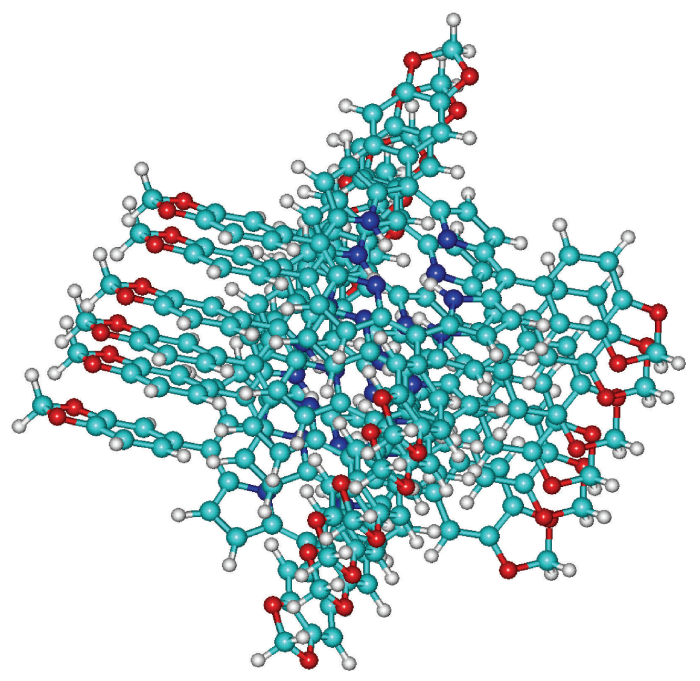

(a)

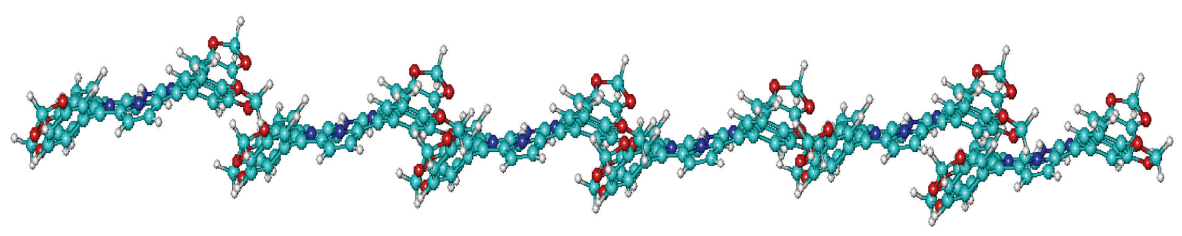

(b)

Figure 1: Face-to-face (a) versus side-by-side (b) aggregates displayed on 5,10,15,20-meso-tetrakis-(3,4-methylenedyoxy)-phenyl-21,23H porphine.

capacity of self-association of this type of molecules are set around spectral analysis as liquid samples [9]. Several parameters, as $\mathrm{pH}$ and solvent polarity, play a major role in aggregation capacity in the liquid state of the sample. By using AFM technique, these influential parameters are excluded. The binomial frame formed by AFM studies and porphyrin structures seem to be lately point of interest, most of them focusing on symmetric structures, free bases [10], from simple compounds [11] to advanced materials [12, 13]. From the behavior of porphyrins bind with peptides [14] to potential sensing devices [15], aspects as supramolecular chirality attract the attention via AFM studies. The variation of peripheral substituents of porphyrins has direct consequences on the formation of porphyrin aggregates, creating high supramolecular chirality targeting supramolecular networks that emulate the biological systems $[16,17]$. One of the most detectable features of the porphyrins is the capacity to aggregate, through several types of interactions, where most of them are governed by the $\pi-\pi$ interactions or hydrogen bonding. This characteristic is desirable for applications in technology, especially in nanotechnology [18]. H-aggregates (face-to-face arrangement) and J-aggregates (side-by-side) are the well-known assemblies as shown in Figure 1, with structures optimized by HyperChem [19]. Instead, for the biological applications as it theranostics is, the capacity to conglomerate is an obvious constraint of the membrane cell penetration process. AFM coupled with some cell lines tests proved to be a useful tool to evaluate the capacity of certain porphyrin to interact with the cell membrane. In this experiment the correlation is made between the scales of magnitude and is not related with the kind of aggregation.

"Scaling" is one of the modern concepts used to characterize the morphology of various complex surfaces, which can be smooth when the "observer" (i.e., the tip in scanning probe microscopy) is far enough and rough when it is close enough. Scaling relations characterize self-similar or self-affine objects and lead to direct computation of an important parameter: fractal dimension [20]. Not all rough surfaces are fractals, but if a surface can be described in terms of fractal geometry, this means that it is self-affine in a statistical way and can be characterized by its fractal dimension. Fractal dimension gives information about the surface roughness, a higher fractal dimension meaning that the surface is very rough, but tells us something about surface geometry; that is, the surface is self-affine. This is a very important thing that when something seems to be disordered and hard to be described, self-affinity and fractal dimension give quantitative information about the surface and describe it. Fractal dimension can be measured in different ways: the direct method implies analysis of topographic images obtained from scanning tunneling microscopy [21-24], scanning probe microscopy [25], or atomic force microscopy [26-28], but there are a lot of other indirect methods: light scattering, neutron scattering, adsorption, and so on. 


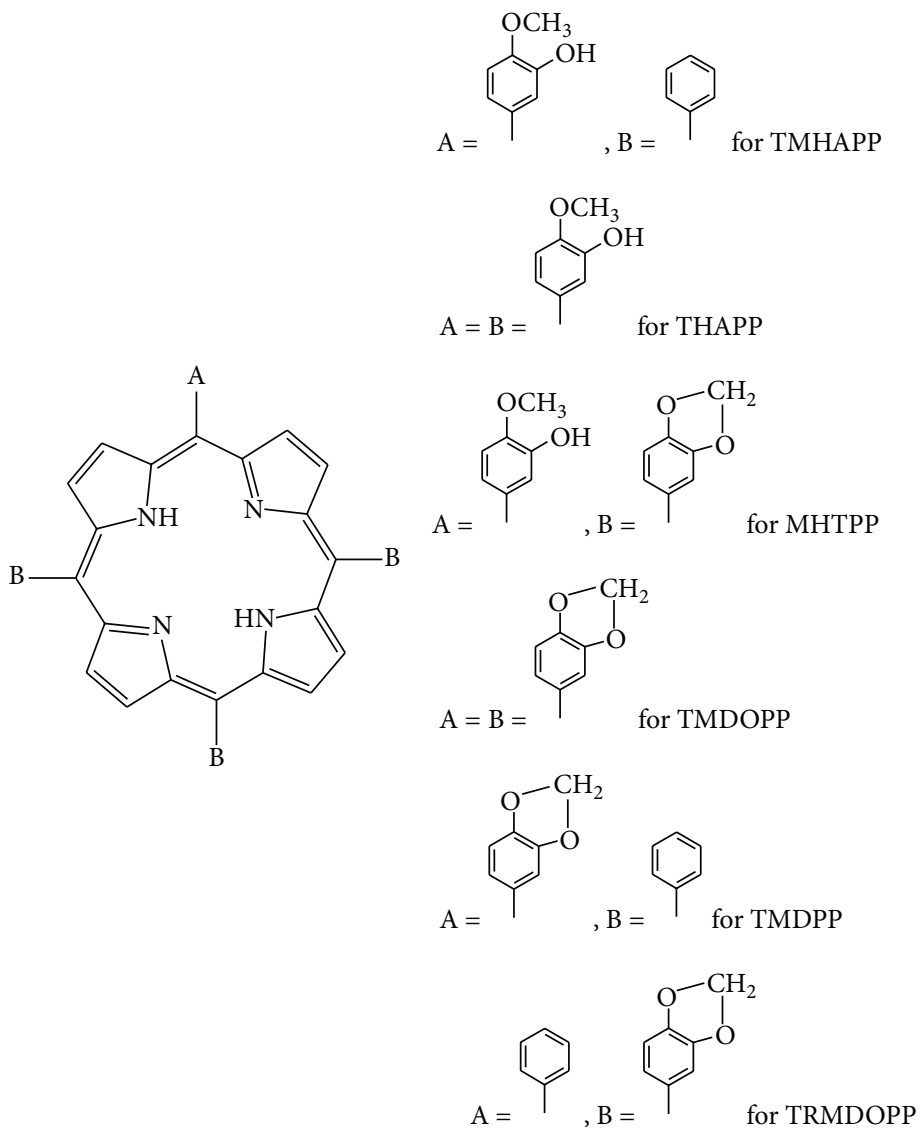

Figure 2: Chemical structures of investigated porphyrins: (1) TMHAPP; (2) THAPP; (3) MHTPP; (4) TMDOPP; (5) TMDPP; and (6) TRMDOPP.

The aim of this paper is to analyze AFM topographic images of asymmetric porphyrins $\left(\mathrm{AB}_{3}\right.$ meso-porphyrinic type structures) in order to analyze their fractal behavior and textural properties and to identify the connection with some results of biological tests. Two mathematical methods were used to compute fractal dimensions: first, the correlation function method [29-31] and, second, the variable length scale method [32]. Our work emphasizes the interdependence between molecular architecture, complex structure analysis, and biological studies, especially AFM technique and porphyrin-cell interaction, in the quest for photosensitizers with improved properties. The order found in their aggregation tendencies is projected in the behavior observed in the cell interaction revealed by cytotoxicity tests.

\section{Experimental Section}

\subsection{Materials and Methods}

2.1.1. Materials. Several asymmetrically substituted porphyrins (Figure 2), 5-(3,4-methylenedyoxy)-phenyl-10,15,20tris-phenyl-21,23H-porphine (TMDPP), 5-(3-hydroxy-4methoxy)-phenyl-10,15,20-tris-phenyl-21,23H-porphine

(TMHAPP), 5-(3-hydroxy-4-methoxy)-phenyl-10,15,20tris-(3,4-methylenedyoxy)-21,23H-porphine (MHTPP), and 5-phenyl-10,15,20-tris-(3,4-methylenedyoxy)-phenyl-21,23H- porphine (TRMDOPP), and symmetrical compounds, 5,10,15,20-meso-tetrakis-(3,4-methylenedyoxy)-phenyl-21, $23 \mathrm{H}$ porphine (TMDOPP) and 5,10,15,20-meso-tetrakis-(3hydroxy-4-methoxy)-phenyl-21,23H-porphine (THAPP), were involved in the present studies.

For the series of $\mathrm{AB}_{3}$ asymmetrical mesoporphyrinic structures, details about synthetic technique are reported elsewhere [33-37]. The substituents attached to the porphyrinic tetrapyrrolic core were chosen so as to balance solubility, aggregation/textural tendencies, high singlet oxygen quantum yield, and cell interaction; two levels of asymmetry occur in the same individual structure: in the macrocyclic core and of the external substituents, complementary to several wellknown "classical" macrocyclic porphyrin-type compounds.

\subsubsection{Methods}

(a) Characterization Methods. Atomic force microscopy (AFM) measurements were carried in true noncontact mode recommended for nondestructive sample scan with a XE-100 apparatus from Park Systems equipped with flexure-guided, cross talk eliminated scanners, using ultrasharp tips $(<8 \mathrm{~nm}$ tip radius; NCHR type from NanosensorsTM) of $125 \mu \mathrm{m}$ length, $30 \mu \mathrm{m}$ width, and $42 \mathrm{~N} / \mathrm{m}$ spring constant $/ \sim 330 \mathrm{kHz}$ resonance frequency. Different imaging scales were used from $8 \times 8 \mu \mathrm{m}^{2}$ to $1 \times 1 \mu \mathrm{m}^{2}$. In order to prepare the 
specimens for AFM investigations, a small quantity of powder was ultrasonically dispersed in ultrapure water (Millie$\mathrm{Q},>18 \mathrm{M} \Omega \mathrm{cm}$ ), thus using the same concentration of the compounds as used in the biological investigations; despite the differences between the nature of solvents used for microscopy and cytotoxicity tests, this part of study chases only the tendencies of aggregation. A drop from this suspension was deposited on atomically flat Highly Oriented Pyrolytic Graphite (HOPG) and dried at room temperature. HOPG was used as to avoid any influence of the substrate on roughness and texture of the investigated samples. The Phase Contrast AFM images were processed by Scanning Probe Image Processor software (SPIPTM v. 4.6.0.0) (SPIP manual available at http://www.imagemet.com/).

(b) Correlation Function Method. A fractal is an object with an observed volume that depends on the resolution (length scale) and follows a power law behavior with a nontrivial exponent over several orders of magnitude. The most important property of fractals is self-similarity, which is the property of a part to look like the whole. Isotropic fractals are self-similar: they are invariant under isotropic scale transformation. When the object scales differently in different space directions, we call it a self-affine fractal. From this point of view, rough surfaces are usually self-affine structures [20]. In our work we shall use to compute fractal dimensions two methods: the height correlation function method $(C)$ and the variable length scale method $(L)$ [32]. Different parameters can be used to characterize the surface roughness. One of these parameters that describe self-affine surfaces is the roughness exponent $\alpha$. In addition to the roughness exponent $\alpha$, it is possible to associate a fractal dimension $D$ with a selfaffine function. The fractal dimension of a self-affine surface can be computed from the height correlation function [2931]:

$$
G(r) \equiv\langle C(\vec{x}, r)\rangle_{x}
$$

where the symbol $\langle\cdots\rangle$ denotes an average over $x$ and $C(\mathbf{x}, r)$ is defined as

$$
C(\vec{x}, r)=[h(\vec{x})-h(\vec{x}+\vec{r})]^{2},
$$

the surface being described by the function $h(\mathbf{x})$ which gives the maximum height of the interface at a position given by $\mathbf{x}$. Thus, the height correlation function $G(r)$ obeys the following scaling relation [29]:

$$
G(r) \sim r^{2 \alpha}, \quad r \ll L,
$$

where, for a surface embedded in a 3-dimensional Euclidean space,

$$
\alpha=3-D
$$

with $D$ being the fractal dimension.

The scaling range in which (3) is obeyed is called the "cut-off" limits and it indicates the range of self-affinity, in other words, the range where there are correlations between the surface points. Correlation function method is suitable for small scaling range because it requires enough points for average in (1), meaning that points computed for low scaling range have low errors and points computed for high scaling range have high errors.

(c) Variable Length Scale Method. The model was proposed by Chauvy et al. [32] and consists of several steps: (i) defining an interval of length $\varepsilon$ (or a box of size $\varepsilon \times \varepsilon$ ); (ii) performing a linear (or planar) least square fit on the data within the interval and calculating the roughness; (iii) moving the interval (box) along the profile (surface) and repeating step (ii); (iv) computing Rms deviation for multiple intervals; and (v) repeating steps (ii)-(iv) for increasing lengths (box sizes). The smallest size for an interval corresponds to 10 data points $(10 \times 10$ points for 3 -dimensional embedded objects $)$ and its maximum size is the total length of the profile (size of the surface). Rms deviation $R_{q \varepsilon}$, averaged over $n_{\varepsilon}$, the number of intervals of length $\varepsilon$, is defined by

$$
R_{q \varepsilon}=\frac{1}{n_{\varepsilon}} \sum_{i=1}^{n_{\varepsilon}} \sqrt{\frac{1}{p_{\varepsilon}} \sum_{j=1}^{p_{\varepsilon}} z_{j}^{2}},
$$

where $z_{j}$ is the $j$ th height variation from the best fit line within the interval $i$ and $p_{\varepsilon}$ is the number of points in the interval $\varepsilon$.

The log-log plot of $R_{q \varepsilon}$ versus $\varepsilon$ gives the Hurst or roughening exponent $H$, and the fractal dimension $D$ can be calculated as

$$
D=D_{T}-H
$$

where $D_{T}$ is the topological dimension of the embedding Euclidean space $\left(D_{T}=2\right.$ for profiles and $D_{T}=3$ for surfaces). Variable length scale method is suitable for higher scaling range compared to correlation function method because the necessity to have enough points in an interval $\varepsilon \times \varepsilon$ to compute Rms deviation $R_{q \varepsilon}$, averaged over $n_{\varepsilon}$, means that $\varepsilon$ must be high enough for a good statistic. Both methods, correlation function method $(C)$ and variable length scale $(L)$ method, will be used to compute fractal dimension of topographic AFM images.

2.2. Preparation Method of Biological Samples. Preliminary in vitro cytotoxicity studies were performed on the Jurkat cell line, human $\mathrm{T}$ cell lymphoblast-like cell line. $10 \mathrm{mM}$ stock solutions of the test porphyrinic compounds were prepared in DMSO by sonication at $22000 \mathrm{~Hz}$ for 30 seconds. For cellular tests stock solutions were further diluted in RPMI 1640 culture medium in the concentration range 1.25$40 \mu \mathrm{M}$. Solutions were handled in sterile conditions. Cell line was cultivated at $37^{\circ} \mathrm{C}$, in $5 \% \mathrm{CO}_{2}$ humid atmosphere, in RPMI 1640 medium supplemented with $100 \mathrm{U} / \mathrm{mL}$ penicillin, $0.1 \mathrm{mg} / \mathrm{mL}$ streptomycin, $0.25 \mu \mathrm{g} / \mathrm{mL}$ amphotericin, $2 \mathrm{mM}$ glutamine, and $10 \%$ fetal bovine serum. For cellular viability and proliferation assays, $5 \times 10^{-4}$ Jurkat cells $/ \mathrm{mL}$ was incubated for $3 \mathrm{~h}$ with various concentrations of compounds. The cellular control consisted in unloaded/untreated Jurkat cells. Cellular viability was assessed by measuring the bioreduction 
of a tetrazolium salt (MTS) to a formazan product, using CellTiter 96 AQeous One Solution Cell Proliferation Assay kit (Promega). The reaction, performed by dehydrogenase enzymes, takes place in metabolically active cells [38] and, therefore, MTS reduction is proportional to the number of viable cells.

Membrane integrity was measured by means of the lactate dehydrogenase (LDH) release assay [39], using CytoTox 96 Non-Radioactive Cytotoxicity Test (Promega). Briefly, cell culture supernatants were collected for the LDH release assay, whilst the rest of the cell suspension was used for the MTS reduction test. Optical densities (OD) were measured for triplicate samples on a Jasco V630 spectrophotometer, in a single beam mode, at $490 \mathrm{~nm}$ without any reference (for LDH release) and at $490 \mathrm{~nm}$ with reference at $640 \mathrm{~nm}$ (for MTS reduction). The mean value of triplicates was calculated and results were further expressed as percent effect relative to control.

\section{Results and Discussion}

3.1. Topography. Typical AFM images for the investigated porphyrins are presented in Figure 3 , at the scale of $2 \times 2 \mu \mathrm{m}^{2}$ for samples (1)-(6), in which the first column represents the 2D topographic images presented in Enhanced Color view mode and the second column the images recorded in Phase Contrast mode, and below them are the characteristic surface profiles (line scans). It was used the Enhanced Color view mode for the topographic $2 \mathrm{D}$ AFM images in order to increase the morphological details of the samples. This is because Enhanced Color uses the change of a pixel relative to its neighbors instead of its absolute value. Phase Contrast working mode was also registered in AFM experiments as to check for possible chemical inhomogeneities of the investigated compounds.

Some topographical characteristics that were consistently observed by AFM are discussed further. (1) TMHAPP is characterized by grains with height up to $10 \mathrm{~nm}$ and typical diameters in the range of $60-100 \mathrm{~nm}$. (2) THAPP shows large grains (agglomerated particles) with diameters from 150 to $400 \mathrm{~nm}$ and height of around $20 \mathrm{~nm}$ (up to 30-40 of the larger ones). Between them, some small surface particles are visible (better in Amplitude or Phase Contrast images), with diameters in the range of $30-40 \mathrm{~nm}$. There is already a visible tendency towards "self-assembling" for sample. (3) MHTPP is in terms of creating large "parcels" with mean height of around $16 \mathrm{~nm}$ and length in the microns range (aspect ratio of up to $1 / 100$ - height/length). (4) TMDOPP exhibit the highest tendency towards agglomeration as could be seen in the corresponding 2D AFM image (topography), 4th row in Figure 3, which could be due to the presence of trace amounts of impurities. (5) TMDPP has also a strong tendency to form large " $2 \mathrm{D}$ " islands of materials (the height of the largest island in the 5 th row being $5 \mathrm{~nm}$ ) instead of stacks as visible for sample (4), TMDOPP. (6) For sample TRMDOPP there are visible some hills beside the formation of large $2 \mathrm{D}$ sheets (few $\mathrm{nm}$ height and few microns length/width), with heights up to $30 \mathrm{~nm}$.
3.2. Fractal Analysis. Fractal dimensions of the porphyrinsbased samples were analyzed (Table 1) using AFM micrographs and (1)-(4) for correlation function method and (5) and (6) for variable length scale method.

From the correlation function method combined with variable length scale method the following fractal characteristics could be summarized. For sample (1) the bare substrate was evidenced $(D=2.16 ; 2.20)$, the porphyrin powder being organized in fractal structures of different values: $2.45-2.46$, for the correlations between the much closed particles (close "neighbors"), and 2.60-2.81, for long distance correlations between porphyrin particles (grains). The structure is self-similar on a rather large domain of selfsimilarity. Regarding sample (2) it is also revealed that the bare substrate is visible in fractal analysis at the scale of $1 \times 1$ and $2 \times 2$ microns $(D=2.00$ and 2.07 , resp.). The porphyrin powder exhibits low distance correlations between particles and low fractal dimensions (2.22-2.30). The image at the scale of $8 \times 8$ microns shows a diffuse structure with fractal dimension of 2.22. Medium and long distance correlations are characterized by fractal dimensions on the range of 2.452.57. Sample (3) has a fractal dimension $D=2.07$ and 2.06 for the bare substrate at the scale of $2 \times 2$ microns while the porphyrin is characterized by self-similar structures of low fractal dimension 2.22-2.37 at low scales of $2 \times 2$ microns (not well agglomerated). A fractal dimension of 2.32 is kept at larger scales on which is superimposed a self-similar columnar structure with $D=2.53-2.60$. In sample (4) selfsimilar agglomerations (aggregates-stacks) with low fractal dimensions of 2.23-2.30 on a large domain of self-similarity are obtained on which a structure with medium fractal dimension (2.51) is superimposed but with a low domain of self-similarity at middle scales. Self-similar columnar structures were obtained for sample (5), with different fractal dimensions, without correlations between structures, though there is a structure of low fractal dimension (2.26) on a large domain of self-similarity. In sample (6), besides the bare substrate $(D=2.18)$, there is an agglomerated selfsimilar structure with low fractal dimension $2.29-2.37$ on a large domain of similarity. A diffuse but self-similar structure, scattered, was also observed ( $D=2.51-2.54)$. The agglomerations are self-correlated with big fractal dimensions, 2.70.

From all the collected data a profile for the aggregation tendencies could be set as follow: TMHAPP $<$ THAPP $<$ MHTPP $<$ TMDOPP $<$ TMDPP $<$ TRMDOPP, the last compound being the most susceptible to form larger aggregates.

3.3. Cytotoxicity Tests. The investigated porphyrinic samples were further tested for their biological response, in two cells interaction tests, eventually targeting the cancer therapy. The results are different, but complementary, as follows: significant influence of aggregation process in the MTS assay results (Figure 4) and constant low impact behavior in $\mathrm{LDH}$ release tests (Figure 5).

3.3.1. MTS Assay. The short-term dark toxicity of the porphyrinic compounds was evaluated on standard $\mathrm{T}$ lymphocytes cell lines Jurkat type. For these biosamples was 


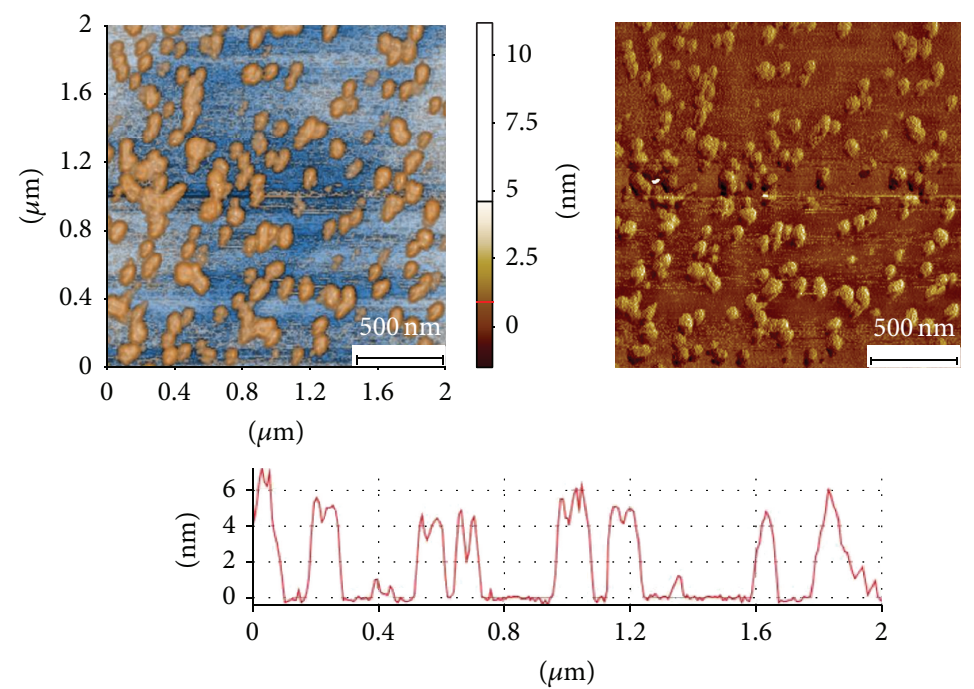

(1)
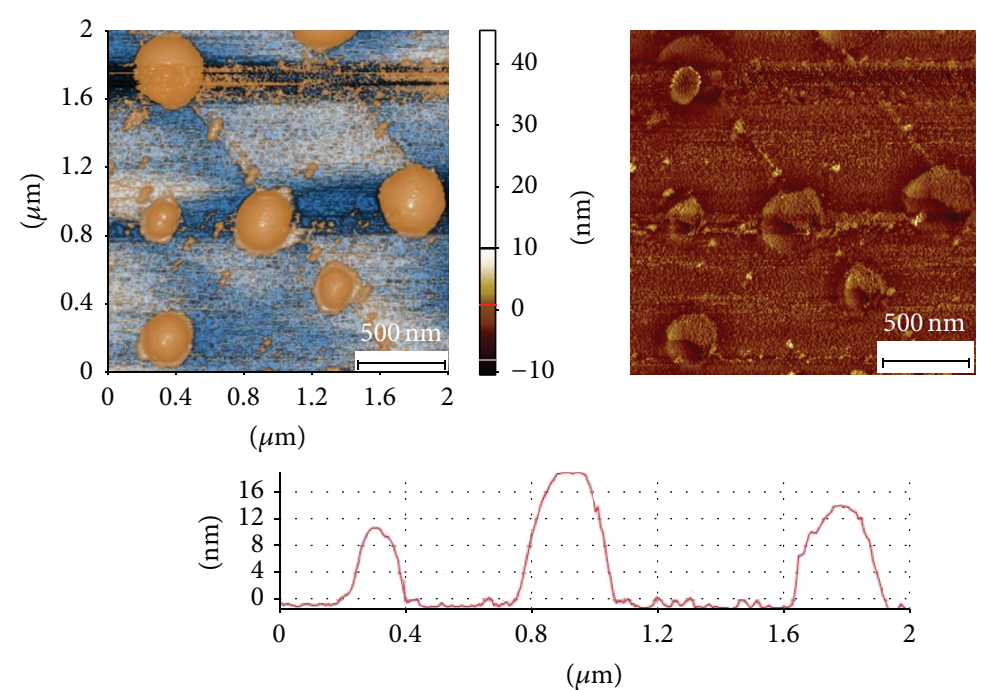

(2)
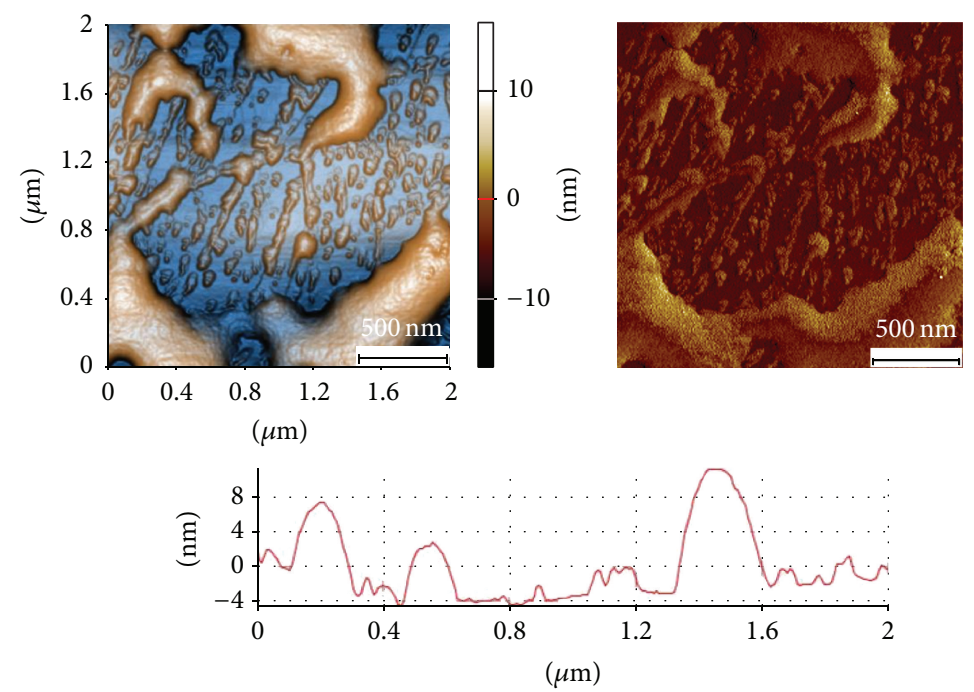

(3)

Figure 3: Continued. 

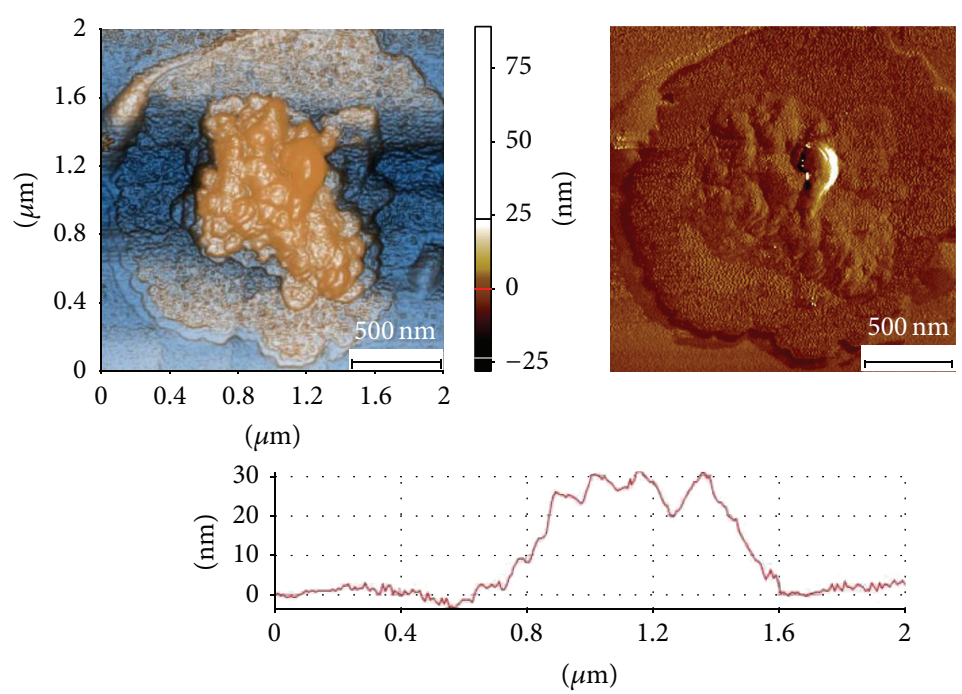

(4)
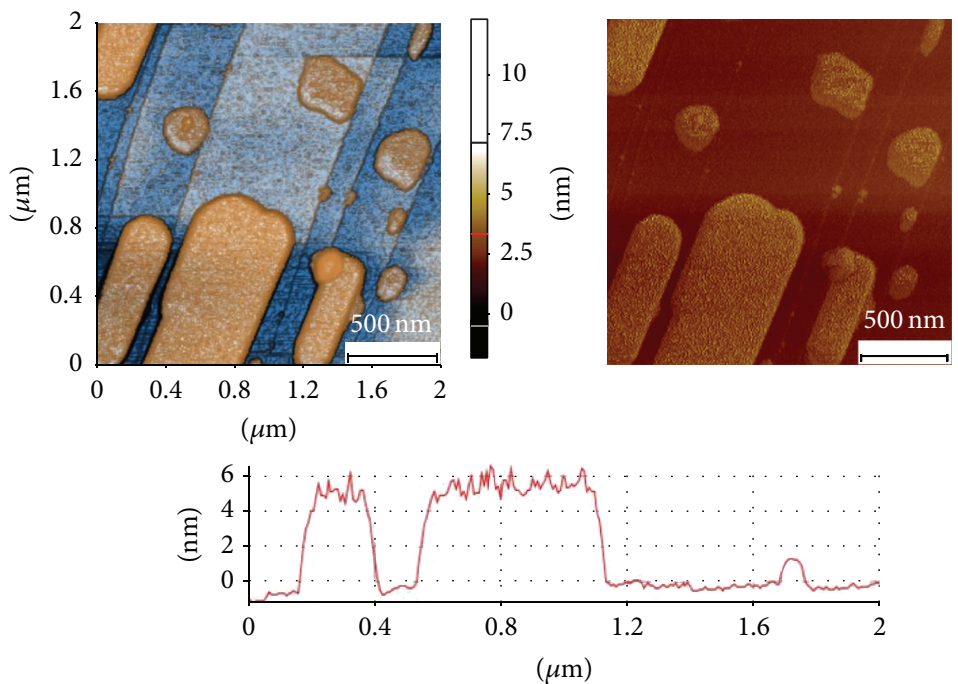

(5)
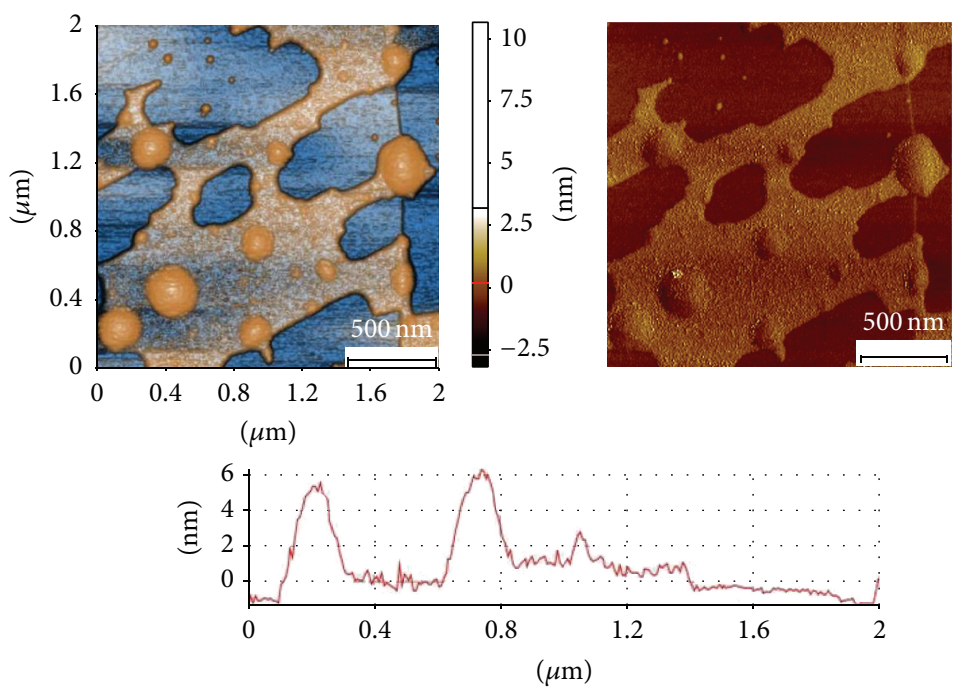

(6)

Figure 3: Typical AFM recorded images at the scale of $(2 \times 2) \mu \mathrm{m}^{2}$ for the investigated porphyrins in which the first column represents the 2D topographic images presented in Enhanced Color view mode and the second column the images recorded in Phase Contrast mode, and below them are the characteristic surface profiles (line scans): (1) TMHAPP; (2) THAPP; (3) MHTPP; (4) TMDOPP; (5) TMDPP; and (6) TRMDOPP 
TABLE 1: Fractal dimensions and self-similarity domains for samples (1)-(6): (1) TMHAPP; (2) THAPP; (3) MHTPP; (4) TMDOPP; (5) TMDPP; and (6) TRMDOPP.

\begin{tabular}{|c|c|c|c|c|}
\hline Sample & Size $(\mu \mathrm{m} \times \mu \mathrm{m})$ & Fractal dimension & Self-similar domain (nm) & Linear correlation coefficient \\
\hline \multirow{3}{*}{ (1) } & $1 \times 1$ & $\begin{array}{l}2.46 \pm 0.01(C) \\
2.16 \pm 0.02(L)\end{array}$ & $\begin{array}{c}19-32 \\
25-103\end{array}$ & $\begin{array}{l}0.997 \\
0.998\end{array}$ \\
\hline & $2 \times 2$ & $\begin{array}{c}2.45 \pm 0.01(C) \\
2.20 \pm 0.02(L) \\
2.81 \pm 0.01(L)\end{array}$ & $\begin{array}{c}23-46 \\
52-129 \\
207-467\end{array}$ & $\begin{array}{l}0.997 \\
0.998 \\
0.966\end{array}$ \\
\hline & $8 \times 8$ & $\begin{array}{c}2.45 \pm 0.03(C) \\
2.71 \pm 0.01(L) \\
2.66 \pm 0.01(L) \\
2.60 \pm 0.02(L)\end{array}$ & $\begin{array}{c}20-88 \\
2182-2597 \\
3012-3948 \\
5714-7272\end{array}$ & $\begin{array}{l}0.972 \\
0.999 \\
0.993 \\
0.947\end{array}$ \\
\hline \multirow{3}{*}{ (2) } & $1 \times 1$ & $\begin{array}{l}2.27 \pm 0.01(C) \\
2.27 \pm 0.01(L) \\
2.00 \pm 0.03(L)\end{array}$ & $\begin{array}{c}22-87 \\
25-64 \\
64-168\end{array}$ & $\begin{array}{l}0.998 \\
0.999 \\
0.991\end{array}$ \\
\hline & $2 \times 2$ & $\begin{array}{l}2.30 \pm 0.01(C) \\
2.45 \pm 0.01(C) \\
2.07 \pm 0.02(L)\end{array}$ & $\begin{array}{c}28-89 \\
89-155 \\
52-363\end{array}$ & $\begin{array}{l}0.997 \\
0.996 \\
0.996\end{array}$ \\
\hline & $8 \times 8$ & $\begin{array}{l}2.57 \pm 0.01(C) \\
2.22 \pm 0.01(L) \\
2.55 \pm 0.02(L)\end{array}$ & $\begin{array}{c}121-973 \\
207-831 \\
831-1558\end{array}$ & $\begin{array}{l}0.997 \\
0.997 \\
0.980\end{array}$ \\
\hline \multirow{3}{*}{ (3) } & $1 \times 1$ & $\begin{array}{l}2.37 \pm 0.01(C) \\
2.53 \pm 0.01(C) \\
2.07 \pm 0.02(L) \\
2.22 \pm 0.02(L)\end{array}$ & $\begin{array}{c}23-80 \\
80-138 \\
52-130 \\
130-259\end{array}$ & $\begin{array}{l}0.995 \\
0.993 \\
0.998 \\
0.995\end{array}$ \\
\hline & $2 \times 2$ & $\begin{array}{l}2.30 \pm 0.01(C) \\
2.06 \pm 0.01(L)\end{array}$ & $\begin{array}{l}22-154 \\
52-363\end{array}$ & $\begin{array}{l}0.996 \\
0.998\end{array}$ \\
\hline & $8 \times 8$ & $\begin{array}{l}2.31 \pm 0.01(C) \\
2.60 \pm 0.01(C) \\
2.32 \pm 0.02(L)\end{array}$ & $\begin{array}{c}58-214 \\
214-403 \\
415-935\end{array}$ & $\begin{array}{l}0.996 \\
0.991 \\
0.996 \\
\end{array}$ \\
\hline \multirow[t]{2}{*}{ (4) } & $2 \times 2$ & $\begin{array}{l}2.30 \pm 0.01(C) \\
2.26 \pm 0.02(L)\end{array}$ & $\begin{array}{l}47-287 \\
52-260\end{array}$ & $\begin{array}{l}0.997 \\
0.997\end{array}$ \\
\hline & $8 \times 8$ & $\begin{array}{l}2.51 \pm 0.01(C) \\
2.23 \pm 0.03(L)\end{array}$ & $\begin{array}{c}133-449 \\
207-1454\end{array}$ & $\begin{array}{l}0.993 \\
0.980\end{array}$ \\
\hline \multirow{2}{*}{ (5) } & $2 \times 2$ & $\begin{array}{l}2.42 \pm 0.01(C) \\
2.55 \pm 0.01(C) \\
2.29 \pm 0.01(L)\end{array}$ & $\begin{array}{c}31-115 \\
115-251 \\
52-363\end{array}$ & $\begin{array}{l}0.998 \\
0.995 \\
0.999\end{array}$ \\
\hline & $8 \times 8$ & $\begin{array}{l}2.36 \pm 0.01(C) \\
2.49 \pm 0.01(C) \\
2.62 \pm 0.01(C) \\
2.26 \pm 0.01(L)\end{array}$ & $\begin{array}{c}20-151 \\
151-300 \\
300-521 \\
208-1143\end{array}$ & $\begin{array}{l}0.996 \\
0.995 \\
0.979 \\
0.997\end{array}$ \\
\hline \multirow{3}{*}{ (6) } & $1 \times 1$ & $\begin{array}{l}2.34 \pm 0.01(C) \\
2.18 \pm 0.02(L)\end{array}$ & $\begin{array}{c}13-35 \\
52-182\end{array}$ & $\begin{array}{l}0.999 \\
0.996\end{array}$ \\
\hline & $2 \times 2$ & $\begin{array}{l}2.37 \pm 0.01(C) \\
2.54 \pm 0.01(C) \\
2.23 \pm 0.01(L)\end{array}$ & $\begin{array}{c}27-81 \\
81-138 \\
52-312\end{array}$ & $\begin{array}{l}0.998 \\
0.993 \\
0.996\end{array}$ \\
\hline & $8 \times 8$ & $\begin{array}{l}2.29 \pm 0.01(C) \\
2.51 \pm 0.01(C) \\
2.70 \pm 0.01(C) \\
2.29 \pm 0.01(L)\end{array}$ & $\begin{array}{c}21-151 \\
151-359 \\
359-676 \\
207-1142\end{array}$ & $\begin{array}{l}0.997 \\
0.997 \\
0.986 \\
0.997\end{array}$ \\
\hline
\end{tabular}

$C$ is correlation function method and $L$ is variable length scale method. 


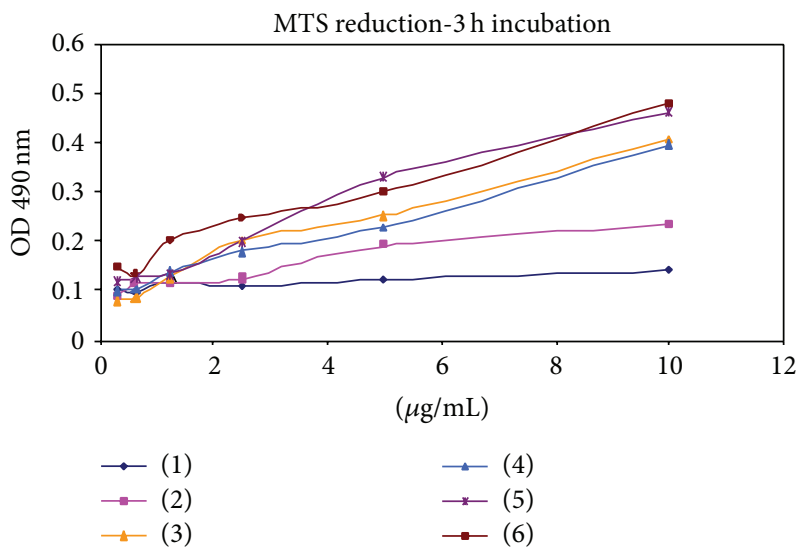

FIGURE 4: MTS assay, on Jurkat cell line, after 3 hours incubation at $37^{\circ} \mathrm{C}$ for samples (1)-(6): (1) TMHAPP; (2) THAPP; (3) MHTPP; (4) TMDOPP; (5) TMDPP; and (6) TRMDOPP.

assessed the cell proliferation from the test of $[3-(4,5-$ dimethylthiazol-2-yl)-5-(3-carboxymethoxyphenyl)-2-(4sulfophenyl)-2H-tetrazolium] reduction, the cells being cultured in 96-well plates in RPMI medium for 3 hours at $37^{\circ} \mathrm{C}$ in an atmosphere containing $5 \% \mathrm{CO}_{2}$ with the porphyrins in DMSO added in different concentrations. The targeted characteristics of porphyrins act invariably for the change of the solvent from water to DMSO.

Also, because the aggregation behavior is largely related to the solvents, the incompatible ones with biological experiments were from start eliminated. Water and DMSO proved to be suitable for this type of studies.

A direct, linear relation between AFM and biological experiments is revealed by the MTS assay studies, establishing a perfect match with the aggregation tendencies in porphyrins:

$$
\begin{aligned}
\text { TMHAPP } & <\text { THAPP }<\text { MHTPP }<\text { TMDOPP } \\
& <\text { TMDPP }<\text { TRMDOPP } .
\end{aligned}
$$

It is observed that, at incipient values of compound concentration, the response in terms of the cell viability remains between restraint borders. Increasing the concentration of added porphyrin solution, the influence in the cell response scattered the compounds behavior; at maximum used concentration the order in response is the same as in the tendency of aggregation scale. The differences in the values of optical density for the MTS release after 3 hours are 5 times bigger for the TRMDOPP than for the TMHAPP. This implies that the porphyrin with lower aggregation tendency behave less aggressive in bio environment.

3.3.2. $L D H$ Release. The cell viability/membrane integrity evaluation was deduced from the lactate dehydrogenase (LDH) release test.

We highlighted that the investigated porphyrinic structures might interfere with cellular LDH particularly after $24 \mathrm{~h}$ incubation, but this issue has to be further investigated; anyway, from cytotoxicity point of view the different aggregation tendencies are not a significant factor in this kind

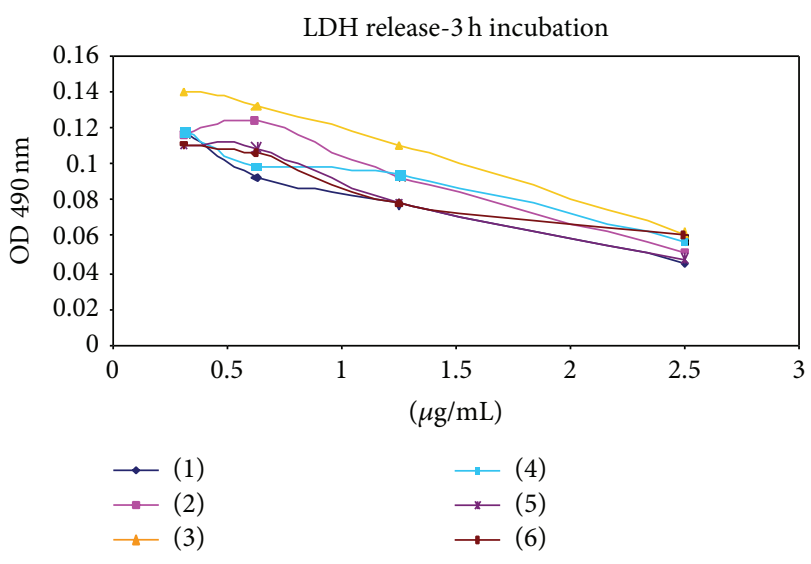

FIGURE 5: LDH release, on Jurkat cell line, after 3 hours incubation at $37^{\circ} \mathrm{C}$ for samples (1)-(6): (1) TMHAPP; (2) THAPP; (3) MHTPP; (4) TMDOPP; (5) TMDPP; and (6) TRMDOPP.

of tests, all the registered values being concentrated in a restraint domain, at lower and increased concentrations. As displayed in Figure 4, in spite of their significant different agglomeration tendencies, the influence on the integrity of cell membrane could be qualified as neutral. The evaluation of the amount of $\mathrm{LDH}$ release, as key index for the permeabilization of plasma membrane in the presence of the porphyrinic samples, leads to the observation that they are nontoxic and, more importantly, independent of the aggregation degree.

Biology tests on these particular compounds are the essential departure milestone in revealing that they localize in the malignant cells and they have a reduced unwanted activity in the absence of laser irradiation.

\section{Conclusions}

$\mathrm{AB}_{3}$ asymmetric meso-porphyrins have been studied by atomic force microscopy in order to evaluate their morphology and establish a direct visual pattern of their tendencies of aggregation; AFM images were further analyzed based on fractal theory with two mathematical methods, namely, the correlation function method and the variable length scale method. From AFM and fractal investigations the following order of the aggregation tendencies of the studied compounds has been established: TMHAPP $<$ THAPP $<$ MHTPP $<$ TMDOPP $<$ TMDPP $<$ TRMDOPP. This order is maintained in the basic biological experiment result involved in the assessment of cell metabolic activity, targeting cancer treatment; the larger the aggregates the lower the power to interfere in the cells activity. Instead, the aggregation degree of porphyrins remains with no consequences when involved in dark-cytotoxicity tests, all proving the same low toxic behavior against tested cells.

\section{Conflict of Interests}

The authors declare that there is no conflict of interests regarding the publication of this paper. 


\section{Acknowledgments}

This research was supported by Project MNT-7-030/2010 of the Romanian Ministry of Education and Research. Support from the EU (ERDF) and Romanian Government infrastructure POS-CCE O 2.2.1 Project INFRANANOCHEM no. $19 / 2009$ is gratefully acknowledged (AFM equipment).

\section{References}

[1] K. Lang, J. Mosinger, and D. M. Wagnerová, "Photophysical properties of porphyrinoid sensitizers non-covalently bound to host molecules; models for photodynamic therapy," Coordination Chemistry Reviews, vol. 248, no. 3-4, pp. 321-350, 2004.

[2] D. M. Guldi, "Fullerene-porphyrin architectures; photosynthetic antenna and reaction center models," Chemical Society Reviews, vol. 31, no. 1, pp. 22-36, 2002.

[3] C. M. Drain, A. Varotto, and I. Radivojevic, "Self-organized porphyrinic materials," Chemical Reviews, vol. 109, no. 5, pp. 16301658, 2009.

[4] M. Ethirajan, Y. Chen, P. Joshi, and R. K. Pandey, "The role of porphyrin chemistry in tumor imaging and photodynamic therapy," Chemical Society Reviews, vol. 40, no. 1, pp. 340-362, 2011.

[5] B. E. Hueger, J. R. Lawter, V. H. Waringrekar, and M. C. Cucolo, US Patent no. 5059619, 1991.

[6] J. S. Lindsey, "Synthetic routes to meso-patterned porphyrins," Accounts of Chemical Research, vol. 43, no. 2, pp. 300-311, 2010.

[7] J. S. Modica-Napolitano, M. Kulawiec, and K. K. Singh, "Mitochondria and human cancer," Current Molecular Medicine, vol. 7, no. 1, pp. 121-131, 2007.

[8] L. Kelbauskas, S. Bagdonas, W. Dietel, and R. Rotomskis, "Excitation relaxation and structure of TPPS $_{4}$ J-aggregates," Journal of Luminescence, vol. 101, no. 4, pp. 253-262, 2003.

[9] A. V. Udal'tsov, A. V. Bolshakova, and J. G. Vos, "Highly ordered surface structure of large-scale porphyrin aggregates assembled from protonated TPP and water," Journal of Molecular Structure, vol. 1065-1066, no. 1, pp. 170-178, 2014.

[10] Y. Zhang, P. Chen, and M. Liu, "A general method for constructing optically active supramolecular assemblies from intrinsically achiral water-insoluble free-base porphyrins," ChemistryA European Journal, vol. 14, no. 6, pp. 1793-1803, 2008.

[11] A. V. Udal'tsov, M. Tosaka, and G. Kaupp, "Microscopy of large-scale porphyrin aggregates formed from protonated TPP dimers in water-organic solutions," Journal of Molecular Structure, vol. 660, no. 1-3, pp. 15-23, 2003.

[12] J. A. A. W. Elemans, R. van Hameren, R. J. M. Nolte, and A. E. Rowan, "Molecular materials by self-assembly of porphyrins, phthalocyanines, and perylenes," Advanced Materials, vol. 18, no. 10, pp. 1251-1266, 2006.

[13] S. Ogi, K. Sugiyasu, S. Manna, S. Samitsu, and M. Takeuchi, "Living supramolecular polymerization realized through a biomimetic approach," Nature Chemistry, vol. 6, no. 3, pp. 188195, 2014.

[14] C. Nakamura, S. Takeda, M. Kageshima et al., "Mechanical force analysis of peptide interactions using atomic force microscopy," Peptide Science, vol. 76, no. 1, pp. 48-54, 2004.

[15] M. Gilaki, "UV-Vis and AFM Study of tetrakis (4-sulfonatophenyl) nano-porphyrin aggregation," Trends in Applied Sciences Research, vol. 6, no. 3, pp. 304-308, 2011.
[16] D. Monti, S. Nardis, M. Stefanelli, R. Paolesse, C. Di Natale, and A. D'Amico, "Porphyrin-based nanostructures for sensing applications," Journal of Sensors, vol. 2009, Article ID 856053, 10 pages, 2009.

[17] D. Monti, M. De Rossi, A. Sorrenti et al., "Supramolecular chirality in solvent-promoted aggregation of amphiphilic porphyrin derivatives: kinetic studies and comparison between solution behavior and solid-state morphology by AFM topography," Chemistry, vol. 16, no. 3, pp. 860-870, 2010.

[18] Z. Wang, C. J. Medforth, and J. A. Shelnutt, "Porphyrin nanotubes by ionic self-assembly," Journal of the American Chemical Society, vol. 126, no. 49, pp. 15954-15955, 2004.

[19] Hypercube Inc, HyperChem Professional 7.51, Hypercube Inc, Gainesville, Fla, USA, 2003.

[20] B. B. Mandelbrot, The Fractal Geometry of Nature, Freeman, New York, NY, USA, 1982.

[21] M. W. Mitchell and D. A. Bonnell, "Quantitative topographic analysis of fractal surfaces by scanning tunneling microscopy," Journal of Materials Research, vol. 5, no. 10, pp. 2244-2254, 1990.

[22] J. P. Carrejo, T. Thundat, L. A. Nagahara, S. M. Lindsay, and A. Majumdar, "Scanning tunneling microscopy investigations of polysilicon films under solution," Journal of Vacuum Science \& Technology B, vol. 9, article 955, 1991.

[23] D. R. Denley, "Scanning tunneling microscopy of rough surfaces," Journal of Vacuum Science \& Technology A: Vacuum, Surfaces, and Films, vol. 8, no. 1, pp. 603-607, 1990.

[24] J. M. Gómez-Rodríguez, A. M. Baró, L. Vázquez, R. C. Salvarezza, J. M. Vara, and A. J. Arvia, "Fractal surfaces of gold and platinum electrodeposits. Dimensionality determination by scanning tunneling microscopy," Journal of Physical Chemistry, vol. 96, no. 1, pp. 347-350, 1992.

[25] J. M. Williams and T. P. Beebe Jr., "Analysis of fractal surfaces using scanning probe microscopy and multiple-image variography. 2. Results on fractal and nonfractal surfaces, observation of fractal crossovers, and comparison with other fractal analysis techniques," Journal of Physical Chemistry, vol. 97, no. 23, pp. 6255-6260, 1993.

[26] G. Dobrescu, C. Obreja, and M. Rusu, "Adhesion AFM applied to lipid monolayers. A fractal analysis," in Fractals: Theory and Applications in Engineering, M. Dekking, J. L. Vehel, E. Lutton, and C. Tricot, Eds., pp. 259-271, Springer, Berlin, Germany, 1999.

[27] G. Dobrescu, C. Obreja, and M. Rusu, "Adhesion AFM applied to DMPE monolayers. A fractal analysis," in Proceedings of the Conference Fractals in Engineering, Delft, The Netherlands, June 1999.

[28] G. Dobrescu, C. Obreja, and M. Rusu, "Fractal analysis of adhesion atomic force microscopy applied to lipid monolayers," Revue Roumaine de Chimie, vol. 43, no. 5, pp. 417-424, 1998.

[29] F. Family, "Dynamic scaling and phase transitions in interface growth," Physica A: Statistical Mechanics and Its Applications, vol. 168, no. 1, pp. 561-580, 1990.

[30] A. L. Barabasi and H. E. Stanley, Fractal Concepts in Surface Growth, Cambridge University Press, Cambridge, UK, 1995.

[31] G. Dobrescu and M. Rusu, "Dynamic scaling method and interface growth," Advances in Colloid and Interface Science, vol. 95, no. 1, pp. 83-93, 2002.

[32] P. F. Chauvy, C. Madore, and D. Landolt, "Variable length scale analysis of surface topography: characterization of titanium surfaces for biomedical applications," Surface and Coatings Technology, vol. 110, no. 1-2, pp. 48-56, 1998. 
[33] R. Socoteanu, "Porphyrinic Compound Double Grafted, Heterocyclic," OSIM Patent No.2008-122035.

[34] R. Socoteanu, "Asymmetrical Substituted Porphyrin Derivative," OSIM Patent No. 2008-122036.

[35] R. Socoteanu, "Asymmetrical Free Base Porphyrin," OSIM Patent No. 2008-122037.

[36] R. Socoteanu, "Porphyrinic Compound as Singlet Oxygen Generator," OSIM Patent No. 2008-122038.

[37] R. Socoteanu, "Asymmetrical Tetrapyrrolic Compound," OSIM Patent no. 2008-122039.

[38] J. A. Barltrop, T. C. Owen, A. H. Cory, and J. G. Cory, "5-(3-carboxymethoxyphenyl)-2-(4,5-dimethylthiazolyl)-3-(4-sulfophenyl)tetrazolium, inner salt (MTS) and related analogs of 3-(4,5dimethylthiazolyl)-2,5-diphenyltetrazolium bromide (MTT) reducing to purple water-soluble formazans as cell-viability indicator," Bioorganic \& Medicinal Chemistry Letters, vol. 1, no. 11, pp. 611-614, 1991.

[39] C. Korzeniewski and D. M. Callewaert, "An enzyme-release assay for natural cytotoxicity," Journal of Immunological Methods, vol. 64, no. 3, pp. 313-320, 1983. 

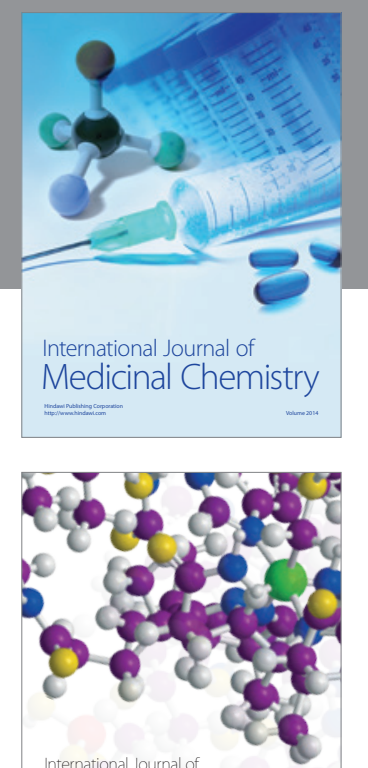

\section{Carbohydrate} Chemistry

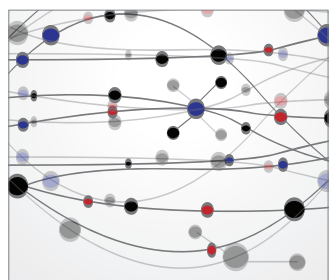

The Scientific World Journal
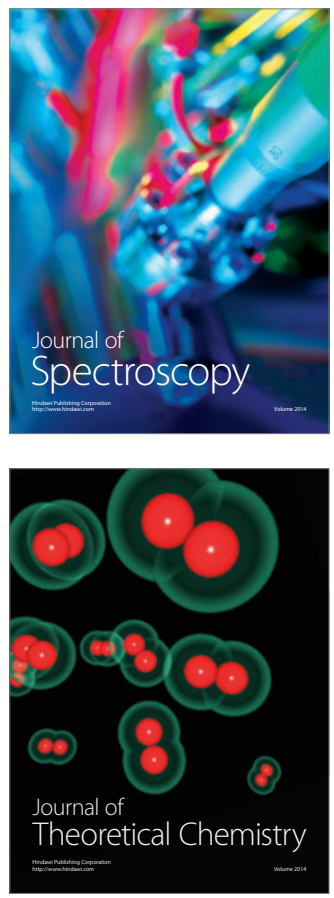
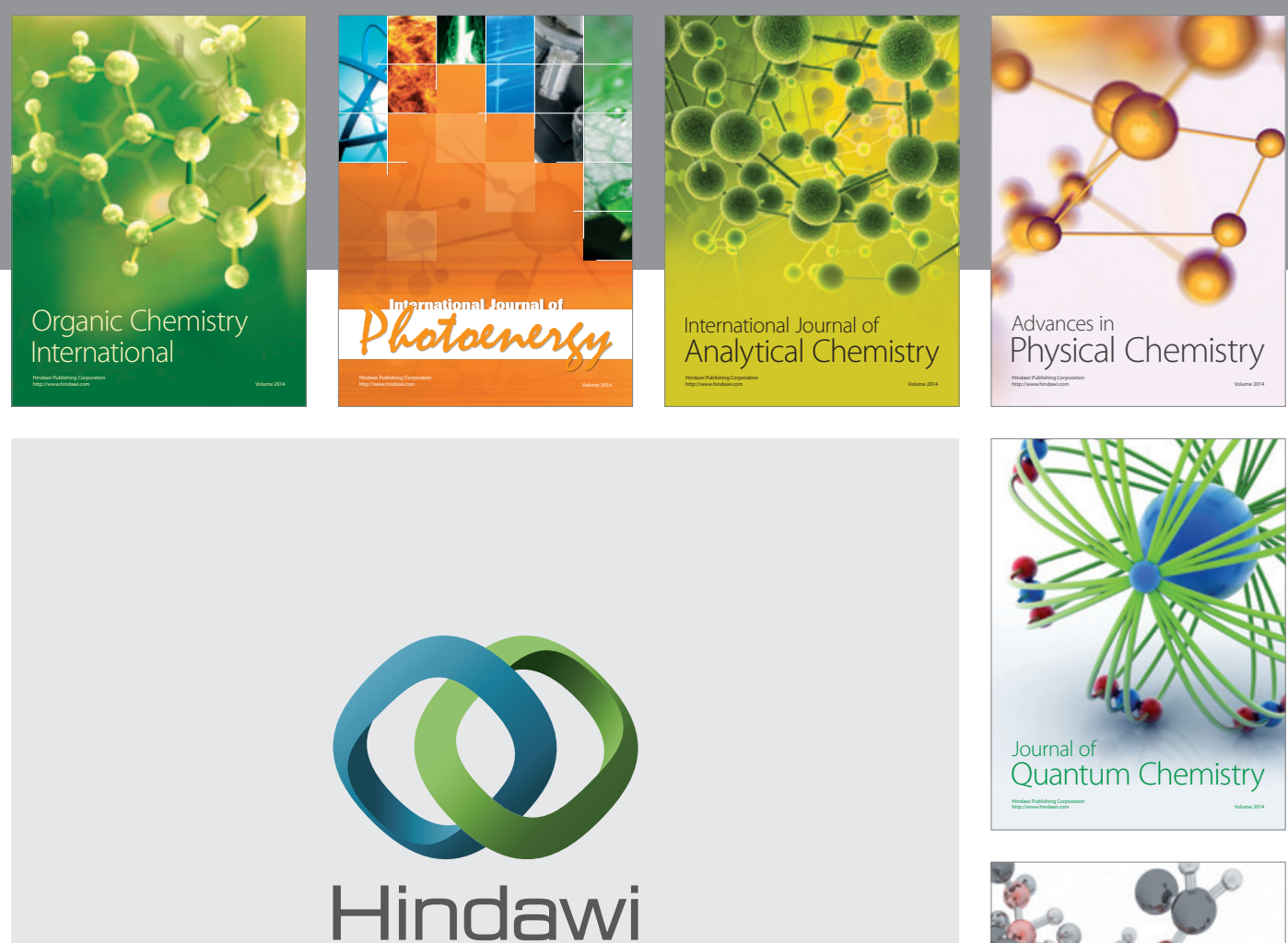

Submit your manuscripts at

http://www.hindawi.com

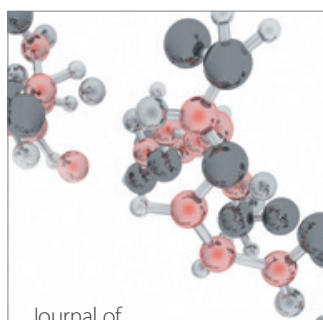

Analytical Methods

in Chemistry

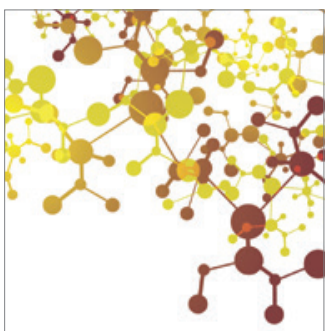

Journal of

Applied Chemistry

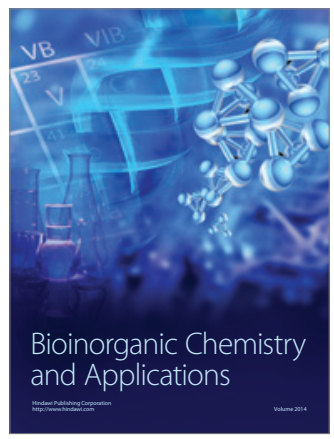

Inorganic Chemistry
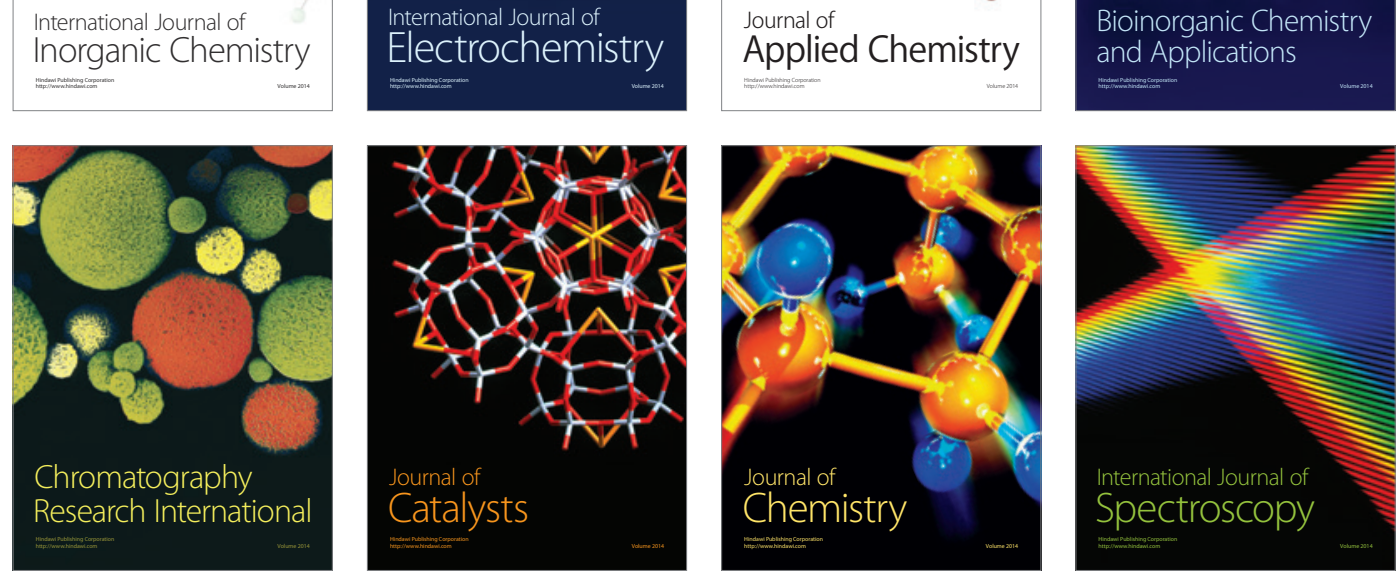